\title{
Are the outcomes of immediate and early single tooth implants comparable to conventionally placed implants?
}

\author{
Abstracted from \\ den Hartog L, Slater JJ, Vissink A, Meijer HJ, Raghoebar GM. \\ Treatment outcome of immediate, early and conventional single-tooth implants in the aesthetic zone: \\ a systematic review of survival, bone level, soft-tissue, aesthetics and patient satisfaction. \\ J Clin Periodontol 2008; 35: 1073-1086 \\ Address for correspondence: $L$ den Hartog, Department of Oral and Maxillofacial Surgery, \\ University Medical Centre Groningen, PO Box 30.001, 9700 RB Groningen, The Netherlands. \\ E-mail: I.den.hartog@kchir.umcg.nl
}

Question: When people require dental implants in the aesthetic zone, is there any difference in the outcome measures between immediate, early and conventionally placed implants?

Data Sources Medline, Embase, the Cochrane Central Register of Controlled Trials (Central) and references of relevant studies were searched. Study selection Longitudinal studies [randomised controlled trials (RCT), clinical trials, cohort-studies and case series] were considered for evaluation. Retrospective studies were excluded. Only case series that investigated at least five patients were considered for inclusion. Outcome measures considered were implant survival, radiographical assessment of marginal peri-implant bone levels, dental-professional-assessed aesthetics, peri-implant biological structures (level of marginal gingiva, papilla index, probing depth, presence of plaque, bleeding on probing), patient satisfaction, and biological and technical complications.

Data extraction and synthesis Articles were screened independently by two examiners and the following parameters recorded: number of patients, implants placed, dropouts, followup time, type of intervention and details of outcomes. To assess the agreement between the two reviewers on the quality of studies, Cohen's kappa statistic was calculated. A meta-analysis was performed using a statistical software package [Comprehensive Meta-analysis Version 2.2, Biostat, Englewood NJ ].

To calculate the overall effects for the included studies, weighted rates together with random effects models were used. Stratification procedures were applied for followup time and type of intervention. No formal heterogeneity testing was conducted.

Results Nineteen studies were included, of which five were RCT, two were clinical trials and 12 were case series. A meta-analysis showed an overall survival rate of $95.5 \% 95 \%$ confidence interval, 93.0-97.1) after 1 year. A stratified meta-analysis revealed no differences in survival between immediate, early and conventional implant strategies. Little marginal peri-implant bone resorption was found together with low incidence of biological and technical complications. No significant differences in outcome measures were reported in clinical trials comparing immediate, early or conventional implant strategies.

Conclusions The selected studies provide promising short-term results for immediate, early and conventional single-implants in the aesthetic zone. Important parameters, such as aesthetic outcome, peri-implant structures and patient satisfaction, however, still need further evaluation. The outcome measures could not be fully evaluated when comparing immediate, early and conventional implants because of the lack of RCT.

\section{Commentary}

The success rate of dental implants in the aesthetic zone is of particular importance to both the general practitioner and the people we treat. Having the option to restore an implant immediately after placement rather than fabricating a transitional removable partial denture, or simply leaving the space edentulous, is of paramount importance to our patients, especially in the aesthetic zone.

In assessing the success rates of immediate, early and conventionally loaded dental implants, a recent study showed that the trends (there were no statistically significant differences) suggest that immediately loaded implants fail more frequently than those that are conventionally loaded, but less often than those loaded early. Therefore, if one wishes to load an implant early, it might be wise to load them immediately (within 1 week) rather than waiting for 1-2 months. ${ }^{1}$

This systematic review evaluated the outcomes of single tooth implants in the aesthetic zone where there were natural adjacent teeth. The investigators then compared immediate (within $48 \mathrm{~h}$ ), early ( $>48 \mathrm{~h}$ but $<3$ months) and conventional (=3 months) loading treatment modalities. Although there are Cochrane reviews evaluating various aspects of dental implants, this systematic review may be considered of special significance because it evaluated those outcomes that are most important to our patients: longevity, aesthetics, and the subject's overall satisfaction with the end result of the treatment.

In terms of longevity, no statistically significant differences in implant survival were found in the clinical trials comparing immediate or early implant procedures with conventional ones. It has been shown that a high degree of implant stability (high value of insertion torque) is one of the prerequisites for a successful immediate/ early loading procedure. ${ }^{2}$

In this systematic review, conclusions could not be drawn in terms of marginal bone changes when comparing the different treatment strategies, but it was shown that with respect to the peri-implant mucosa, the clinical crown height was acceptable in significantly more cases in the early placement groups than in the conventional groups. Although these clinical crown height results were based on only one study that was included in the review, one could reasonably assume that maintaining a clinical crown height that is closer to the "Golden Proportion" would lead to a more ideal aesthetic outcome and therefore higher patient satisfaction.

Even though reported satisfaction levels were high, only four of the studies in this systematic review evaluated this outcome. It is possible however, to suggest that implants placed with the imme- 
ORAL SURGERY

diate or early implant protocol might provide higher patient satisfaction and aesthetic outcomes than the conventional approach, possibly because of the preservation of the alveolar ridge. ${ }^{3}$

Although strong conclusions could not be drawn about which loading option is the overall treatment strategy of choice, and the fact that there needs to be more long-term research in respect to aesthetic outcomes and patient satisfaction, research tends to suggest that immediate placement and loading of dental implants could lead to a more satisfying experience for the patient, a better aesthetic outcome, and little added risk in terms of implant survival.

Gary L Stafford

Department of General Dental Sciences, Marquette University School of Dentistry, Milwaukee, Wisconsin, USA

1. Esposito M, Grusovin MG, Achille H, Coulthard Paul, Worthington HV. Interventions for replacing missing teeth: different times for loading dental implants. Cochrane Database Syst Rev 2009; issue 1.

2. Esposito M, Grusovin MG, Martinis E, Coulthard P, Worthington HV. The effectiveness of immediate, early, and conventional loading of dental implants: a Cochrane

\section{Practice points}

- It is possible to successfully load dental implants immediately or early after their placement in selected patients, but careful patient selection and treatment planning should precede this modality of treatment.

- There is some indication that there is a correlation between aesthetic appearance before implant treatment and the final aesthetic result from both patients' and the clinicians' perspectives. There are doubts however, as to whether or not this is true for patient satisfaction. Patients who have a poor pre-operative condition might have a different attitude when comparing themselves against those that have a more ideal starting point.

systematic review of randomised controlled clinical trials. Int / Oral Maxillofac Implants 2007: 22: 893-904

3. Esposito M, Grusovin MG, Koukoulopoulou A, Coulthard P, Worthington HV.

Interventions for replacing missing teeth: dental implants in fresh extraction sockets (immediate, immediate-delayed and delayed implants). Cochrane Database Syst Rev 2006; issue 4.

Evidence-Based Dentistry (2009) 10, 77-78. doi:10.1038/sj.ebd.6400669 